\title{
A SOCIEDADE DA INFORMAÇÃO E DO CONHECIMENTO E OS ESTADOS BRASILEIROS
}

\section{LA SOCIEDAD DE LA INFORMACIÓN Y EL CONOCIMIENTO Y LOS ESTADOS DE BRASIL}

Cecília Araújo Rabelo Corrêa - cissa_0903@yahoo.com.br Mestre em Administração Pública pela Fundação João Pinheiro (FJP/CPA).

Elisa Maria Pinto da Rocha - elisa.rocha@fjp.mg.gov.br Doutora Ciência da Informação pela Universidade Federal de Minas Gerais (UFMG). Diretora do Centro de Pesquisas Aplicadas da Fundação João Pinheiro (FJP/CPA).

Jane Noronha Carvalhais - teianc@gmail.com Doutora em Ciências Sociais pela Pontifícia Universidade Católica de Minas Gerais (PUCMinas). Docente da Fundação Educacional de Divinópolis (FUNEDI/UEMG).

Simone Cristina Dufloth - sduf@uol.com.br Doutora em Ciência da Informação pela Universidade Federal de Minas Gerais (UFMG). Pesquisadora da Fundação João Pinheiro (FJP/CPA).

\section{RESUMO}

Introdução: A expressão Sociedade da Informação e do Conhecimento é utilizada para caracterizar o novo padrão de acumulação capitalista que assume maior relevância a partir dos anos 1990. Nesse novo padrão tecnológico e produtivo, a informação, conhecimento e as inovações tecnológicas passam a ser fatores determinantes do desenvolvimento socioeconômico de uma nação. O Brasil possui grande desigualdade interna o que tende a influenciar as condições de inserção dos estados brasileiros nesta Sociedade.

Objetivo: O objetivo deste artigo é analisar as condições de inserção dos estados brasileiros no padrão tecno-produtivo que ora se configura no Brasil e no mundo.

Metodologia: Revisão de Literatura e Análise Documental. 
Cecília Araújo Rabelo Corrêa; Elisa Maria Pinto da Rocha; Jane Noronha Carvalhais;

Simone Cristina Dufloth

A sociedade da informação e do conhecimento e os estados brasileiros

Resultados: Os resultados corroboram a ideia geral acerca da heterogeneidade das condições de inserção dos estados no novo padrão tecnológico e econômico. A grande maioria dos estados apresentou condições de inserção desfavoráveis, e as dimensões que explicam esta posição desconfortável são: Infraestrutura, Acesso e Uso das Tecnologias da Informação e das Comunicações, e Habilidades e Disponibilidade de Recursos Humanos.

Conclusões: $O$ estudo sugere que avanços na construção de condições favoráveis de inserção dos estados dependem fundamentalmente, da adoção de medidas capazes de aprofundar a sinergia entre a política de ciência e tecnologia, a política educacional e industrial.

Palavras-chaves: Sociedade da informação e do conhecimento. Condições de inserção dos estados. Indicadores de mensuração.

\section{INTRODUÇÃO}

Os avanços e o aumento da velocidade de disseminação das Tecnologias de Informação e das Comunicações (TIC) resultaram em profundas mudanças sociais, econômicas nos países, e para designar o momento que emerge deste contexto de transformações utiliza-se a expressão Sociedade da Informação e do Conhecimento $(\mathrm{SIC})$.

Sob a perspectiva da consolidação da Sociedade da Informação e do Conhecimento, a informação, o conhecimento e as inovações tecnológicas passam a ser fatores determinantes do desenvolvimento socioeconômico de países, regiões e empresas. Uma vez que mesmo internamente os países possuem diferenciações quanto à inclusão digital, o processo de inserção, em decorrência, tende a não ser linear.

O processo de transição rumo à SIC é global e heterogêneo (LUGONES, 2002) e o conhecimento desta realidade é essencial para traçar políticas voltadas para a promoção de ambiente favorável à inserção de países e regiões no novo padrão tecnoeconômico de produção denominado Sociedade da Informação e das Comunicações. Não apenas no Brasil, como também no conjunto dos países da América Latina, são ainda recentes e em decorrência, incipientes, as discussões sobre as características que vem assumindo o processo de formação da SIC, ou seja, o novo padrão de produção. Estudos sobre o tema podem se constituir subsídio fundamental para países e regiões que preocupados em aproveitar 
Cecília Araújo Rabelo Corrêa; Elisa Maria Pinto da Rocha; Jane Noronha Carvalhais;

Simone Cristina Dufloth

A sociedade da informação e do conhecimento e os estados brasileiros

eventuais benefícios advindos do novo contexto, aumentando seus ganhos na economia global com maior competitividade.

As unidades da federação brasileira são marcadas por desigualdades no desenvolvimento socioeconômico, o que tende a influenciar o quadro existente de condições de inserção na SIC. Além disto, a realidade dos estados em termos de aspectos tais como: infraestrutura tecnológica, acesso e uso das novas tecnologias da informação e das comunicações, disponibilidade de mão de obra qualificada, e priorização de gastos públicos na área de desenvolvimento científico e tecnológico, impõe necessidade de políticas públicas que amenizem as desigualdades existentes, especificamente as desigualdades digitais entre os estados.

Este trabalho parte da premissa de que as condições de inserção dos estados na SIC apresentam-se heterogêneas, e nesse sentido, a preocupação central que orienta a elaboração da pesquisa pode ser retratada pelos seguintes questionamentos: Os estados brasileiros reúnem condições que favorecem a sua inserção na Sociedade da Informação e do Conhecimento? Quais as principais fragilidades que se apresentam a este processo de inserção?

O objetivo do trabalho é analisar as condições de inserção dos estados brasileiros no novo padrão tecnoeconômico, buscando-se inferir sobre as fragilidades ou pontos fracos que se impõem a este processo no âmbito das unidades da federação. Ao ampliar informações e conhecimentos sobre as características que este processo assume nos estados da federação, esta pesquisa contribui para subsidiar a formulação de políticas públicas voltadas para o desenvolvimento de uma efetiva Sociedade da Informação e do Conhecimento no Brasil.

O artigo encontra-se estruturado em cinco seções, além desta Introdução. $\mathrm{Na}$ segunda seção, elabora-se referencial teórico sobre o surgimento e características da SIC, e sobre iniciativas de mensuração de aspectos relevantes da SIC nos países da América Latina, enfatizando-se o Manual de Lisboa (LUGONES; SUÁREZ; ALVES, 2009). Após, é descrito o tratamento metodológico utilizado para a construção da ferramenta de análise, qual seja o Índice das Condições de Inserção dos Estados Brasileiros na Sociedade da Informação e do Conhecimento (IISIC-E). A análise dos principais resultados do trabalho é apresentada na quarta seção, e por fim, são elaboradas as considerações finais acerca do estudo. 
Cecília Araújo Rabelo Corrêa; Elisa Maria Pinto da Rocha; Jane Noronha Carvalhais;

Simone Cristina Dufloth

A sociedade da informação e do conhecimento e os estados brasileiros

\section{ESTADO DA ARTE: SOCIEDADE DA INFORMAÇÃO E DO CONHECIMENTO E AS INICIATIVAS VOLTADAS PARA A SUA MENSURAÇÃO}

As sociedades contemporâneas vêm passando por transformações na dinâmica tecnológica, fenômeno este que se acentua a partir dos anos iniciais do século XXI. Os avanços nas áreas de informática, telecomunicações e microeletrônica influenciam na velocidade de disseminação das tecnologias da informação, especialmente por meio do computador, internet, equipamentos e aparelhos de telecomunicações.

Segundo Lugones (2002, p. 4), entre: "[...] as tecnologias da informação e das comunicações (TIC) incluem tecnologias maduras como o radio, a telefonia fixa, televisão, como também tecnologias mais dinâmicas, como a informática, a transmissão de dados via satélite e fibra ótica, a telefonia celular e a internet."

O processo de disseminação das novas tecnologias provoca mudanças na sociedade, tanto nas dimensões tecnológica e econômica quanto nos aspectos socioculturais, políticos e institucionais. A este cenário de transformação da sociedade que emerge nos anos 90 atribui-se a expressão Sociedade da Informação e do Conhecimento (SIC). O contexto econômico marcado pela revolução tecnológica, por sua vez, é retratado pela expressão Economia Informacional (ROCHA, 2003).

De acordo com Míssio (2007):

[...] o conceito Sociedade da Informação compreende a sistematização de um conjunto de transformações científicas e tecnológicas impulsionadas pelo desenvolvimento da microeletrônica, da informática e de suas tecnologias associadas, notadamente a partir do desencadeamento da Segunda Guerra Mundial (apud FUNDAÇÃO JOÃO PINHEIRO, 2010, p. 11).

Em interessante pesquisa sobre o tema, a Fundação João Pinheiro aponta as algumas das principais características do novo padrão tecnoeconômico de produção: (a) crescente complexidade das novas tecnologias, crescentemente mais intensivas em informação e conhecimento; (b) aceleração do processo de geração de novos conhecimentos e de difusão de inovações; (c) novos métodos de pesquisa 
Cecília Araújo Rabelo Corrêa; Elisa Maria Pinto da Rocha; Jane Noronha Carvalhais;

Simone Cristina Dufloth

A sociedade da informação e do conhecimento e os estados brasileiros

e desenvolvimento, em que os sistemas de base eletrônica assumem papéis cada vez mais importantes tanto na geração de novos conhecimentos como na aquisição de conhecimentos já existentes e desenvolvimento de novas configurações; (d) aprofundamento do nível de conhecimentos tácitos (não codificáveis e específicos de cada unidade produtiva e de seu ambiente), resultando na necessidade de investimentos nas atividades de treinamento e qualificação de recursos humanos; (e) mudanças profundas na forma de gestão e de organização das empresas no sentido de permitir maior flexibilidade e integração de suas funções (pesquisa, produção, administração, marketing etc.), bem como de ampliar os níveis de interligação com outros atores (usuários, produtores, fornecedores e prestadores de serviços, outras organizações); e (f) exigência de novas estratégias, políticas e formas de intervenção governamental, para forjar a consolidação de um sistema de ciência, tecnologia e inovação (CT\&l) eficiente e maduro (FUNDAÇÃO JOÃO PINHEIRO, 2010).

Sob a perspectiva da Economia Informacional, embora a velocidade da internacionalização do capital financeiro iniciado com a Revolução Industrial tenha aumentado significativamente no passado recente - fazendo com que, a princípio possa se pensar em uma configuração totalmente nova da Sociedade da Informação e do Conhecimento que emerge neste cenário - vale ressaltar que o pressuposto do capitalismo continua se mantendo na Economia Informacional, qual seja: o estabelecimento de relações de produção orientadas para a maximização do lucro.

O novo contexto da SIC e da Economia Informacional influencia o desenvolvimento social e econômico de países e regiões, podendo-se dizer que o nível de desenvolvimento é influenciado e sofre a influência das transformações decorrentes da disseminação das novas tecnologias. Dessa forma, o acesso e uso de tecnologias podem representar vantagens ou desvantagens para 0 desenvolvimento socioeconômico.

De modo geral, as diferenciações existentes nas condições de acesso e uso das tecnologias da informação e das comunicações são entendidas como "brechas digitais" e configuram um desafio a ser enfrentado por países e regiões. Inserir-se na Sociedade da Informação e do Conhecimento, entretanto, requer não apenas a existência de infraestrutura tecnológica adequada e suficiente para promover 0 acesso e o uso das novas tecnologias, como também e principalmente, requer a 
Cecília Araújo Rabelo Corrêa; Elisa Maria Pinto da Rocha; Jane Noronha Carvalhais;

Simone Cristina Dufloth

A sociedade da informação e do conhecimento e os estados brasileiros

existência de recursos humanos que detenham níveis de qualificação compatíveis com os requerimentos do novo padrão tecnoeconômico, e a alocação prioritária de recursos públicos na promoção do desenvolvimento científico e tecnológico. Ou seja, inserir-se na SIC torna-se uma estratégia de desenvolvimento socioeconômico das nações.

A constatação de que as tecnologias perfazem condição para a participação dos países na economia globalizada levou os países a formularem e adotarem políticas públicas como meio para construir a SIC. Os governos passaram a desenvolver políticas públicas no sentido de habilitar e capacitar os diversos segmentos da sociedade para que, de fato possam usufruir dos benefícios e oportunidades por ela propiciados.

Em termos gerais, pode-se dizer que o escopo que as políticas públicas voltadas para a consolidação da SIC assumem nos distintos países guarda relação com seu nível de desenvolvimento socioeconômico. Assim, o desenho e a implementação de programas e projetos públicos de promoção de um ambiente favorável ao processo de inserção pode apresentar especificidades e particularidades próprias. O Brasil caracteriza-se pela existência de grande desigualdade interna entre regiões e estados, e o estudo das condições de inserção dos estados na SIC mostra-se bastante importante enquanto subsídio ao processo de formulação de políticas públicas compatíveis com as realidades regionais e estaduais.

Os índices ou indicadores sintéticos são instrumentos que podem ser utilizados para conhecer melhor determinado fenômeno ou realidade, como é o caso da Sociedade da Informação e do Conhecimento ora em configuração no Brasil. Ou seja, os indicadores são peças de informação que ampliam a comunicação e a compreensão de determinado fenômeno. De acordo com Scandar Neto, Jannuzzi e Silva (2008, p. 7) os indicadores sintéticos buscam "apreender a realidade social através de uma única medida, resultante da combinação de múltiplas medições das suas dimensões analíticas quantificáveis".

As experiências de mensuração do processo de desenvolvimento e consolidação da Sociedade da Informação possuem caráter ainda preliminar e no Brasil, análises desagregadas por estado são ainda inexistentes. Ampliar o conhecimento a este respeito torna-se importante e as principais iniciativas 
Cecília Araújo Rabelo Corrêa; Elisa Maria Pinto da Rocha; Jane Noronha Carvalhais;

Simone Cristina Dufloth

A sociedade da informação e do conhecimento e os estados brasileiros

internacionais neste campo evidenciam ser preciso conhecer a realidade regional e local no sentido de gerar informações que subsidiem a formulação de políticas públicas direcionadas para as especificidades regionais e locais. No caso de países caracterizados por grande desigualdade interna - como é o caso do Brasil - esta constatação mostra-se ainda mais relevante.

O Manual de Lisboa (LUGONES; SUÁREZ; ALVES, 2009) se constitui na mais completa e interessante iniciativa voltada para a mensuração da Sociedade da Informação nos países da América Latina. As sugestões nele contidas avançam para uma caracterização mais complexa do fenômeno tratado, também, neste artigo: a transição dos países para a Sociedade da Informação.

O núcleo central da proposta do Manual de Lisboa é entender a SIC a partir de uma matriz de indicadores que representa conceitos e sugere a obtenção de informações relevantes, e cujo objetivo é sintetizar os elementos necessários à compreensão da emergência da SIC.

"A sua intenção é contribuir para a análise integral do fenômeno da Sociedade do Conhecimento e é por isso que se apresenta como uma ferramenta para o estudo, a interpretação e a análise dos indicadores gerados." (LUGONES; SUÁREZ; ALVES, 2009, p. 1).

A matriz de indicadores do Manual de Lisboa (LUGONES; SUÁREZ; ALVES, 2009), mostrada na Figura 1, evidencia que a configuração da SIC é influenciada diretamente pelo nível de desenvolvimento alcançado por quatro setores básicos: educação, ciência e tecnologia, informática e serviços de alto valor agregado, e telecomunicações. Estes setores representam as atividades básicas sobre as quais os agentes tomam decisões e adquirem ferramentas tecnológicas.

Sob a perspectiva do Manual de Lisboa,

Estes setores constituem a moldura dentro da qual os agentes e atores sociais buscam, de um lado, aproveitar, da forma mais favorável e simples possível, as ferramentas disponíveis para criar e gerir a informação; e de outro, produzir e ofertar, de maneira crescente, os bens e serviços intensivos em conhecimento (LUGONES; SUÁREZ; ALVES, 2009, p. 1).

O nível de desenvolvimento dos setores ou atividades básicas é interpretado como condição necessária, mas não suficiente para o desenvolvimento da Sociedade da Informação. Por exemplo, se o nível de desenvolvimento desses 
Cecília Araújo Rabelo Corrêa; Elisa Maria Pinto da Rocha; Jane Noronha Carvalhais;

setores for baixo, os agentes terão maior dificuldade para assimilar as práticas e ferramentas tecnológicas característicos da Sociedade da Informação.

Os agentes e as variáveis teóricas são representados na sub-matriz (linhas). No caso dos agentes, o Manual sugere as seguintes categorias: empresas, agregados familiares, governo e outras instituições. Estas categorias são classificadas de acordo As quatro categorias de agentes sociais e econômicos são classificadas de acordo com a motivação que utilizam a informação, o conhecimento e as tecnologias da informação e das comunicações.

Figura 1 - Matriz de Indicadores proposta no Manual de Lisboa para a Mensuração da Transição dos Países em Direção à Sociedade da Informação.

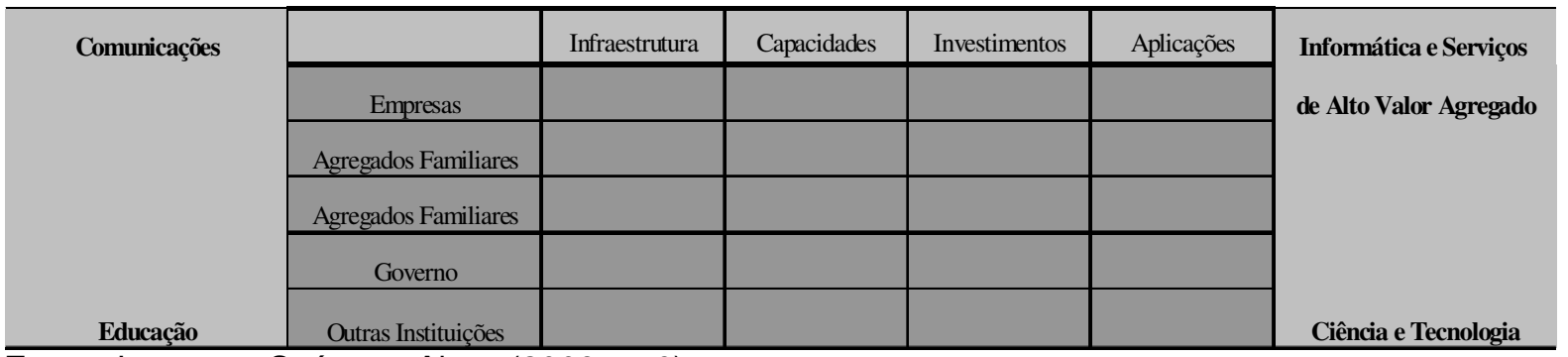

Fonte: Lugones, Suárez e Alves (2009, p. 6).

Os quatro eixos temáticos, por sua vez, podem ser divididos em dois grupos: Infraestrutura e Capacidades; e Investimentos e Aplicações. Os dois primeiros registram informações sobre os recursos físicos e humanos que os agentes possuem. Os eixos Investimentos e Aplicações correspondem a ações que contribuem para a melhoria dos recursos utilizados e captam tendência de inserção ou distanciamento dos países em relação à Sociedade da Informação.

A matriz permite visualizar dezesseis possibilidades de interseções para as quais são criados indicadores a partir das bases estatísticas disponíveis nos países. Estas possibilidades sintetizam os principais aspectos a serem enfatizados no processo de transição dos países para a Sociedade da Informação. Assim, o uso da matriz como ferramenta de análise possibilita uma abordagem dinâmica sobre o processo de transição dos países da América Latina para a Sociedade da Informação.

Conforme destacado no Manual de Lisboa, os indicadores devem possuir significado relevante para os países da América Latina, e simultaneamente, possibilitar comparações internacionais. Os indicadores que buscam retratar a 
Cecília Araújo Rabelo Corrêa; Elisa Maria Pinto da Rocha; Jane Noronha Carvalhais;

Simone Cristina Dufloth

A sociedade da informação e do conhecimento e os estados brasileiros

Sociedade da Informação devem apontar caminhos a serem trilhados pelos países da região rumo à sua inserção no novo padrão tecnológico e produtivo, ou seja, na Sociedade da Informação. Assim, é importante que os indicadores sejam capazes de refletir fragilidades e potencialidades dos países latino-americanos e que subsidie a formulação de políticas públicas que amenizem as desigualdades inter e intraregionais.

A desigualdade regional existente entre os estados brasileiros no plano econômico e social se reflete também, na esfera da inclusão digital. O Mapa da Exclusão Digital (NERI, 2003) elaborado pela Fundação Getúlio Vargas demonstrou que apenas $8,31 \%$ da população brasileira estava conectada à internet, e evidenciou a grande diferença digital existente entre as zonas urbana e rural, bem como entre as regiões mais ricas e mais pobres do País.

Os principais obstáculos existentes no Brasil e que restringem o acesso da população às novas tecnologias relacionam-se à carência da infraestrutura tecnológica, além de limitações associadas ao setor educacional e dos altos custos de acesso da população às novas tecnologias (CENTRO DE ESTUDOS SOBRE AS TECNOLOGIAS DA INFORMAÇÃO E_DA COMUNICAÇÃO, 2010).

No que diz respeito a comparações internacionais, estudos como o de Weiselfisz (2007), revelou que a proporção de brasileiros que possuía acesso à internet em 2001 era 8,3\%, percentual este que avançou para 13,6\% em 2005. Em termos de acesso à internet, os dados indicaram que o Brasil encontrava-se em posição menos favorável do que outros países latino-americanos, tais como: Chile, Costa Rica, Uruguai e Argentina, em 2005.

No caso brasileiro, as distorções internas são muitas vezes ainda maiores do que as aquelas registradas entre o Brasil e outros países da América Latina. Assim é que no Distrito Federal, $41,1 \%$ da população utilizou a internet nos últimos três meses, enquanto que no Estado de Alagoas esta proporção foi 7,6\%, de acordo com dados da PNAD 2005. Levantamentos do CETIC.br na pesquisa TIC Domicílios evidencia a desigualdade existente entre as regiões brasileiras, utilizando dados mais recentes (Gráfico 1). 
Cecília Araújo Rabelo Corrêa; Elisa Maria Pinto da Rocha; Jane Noronha Carvalhais;

Gráfico 1 - Proporção de Domicílios Urbanos com Acesso à Internet de 2005 a 2010 $(\%)$

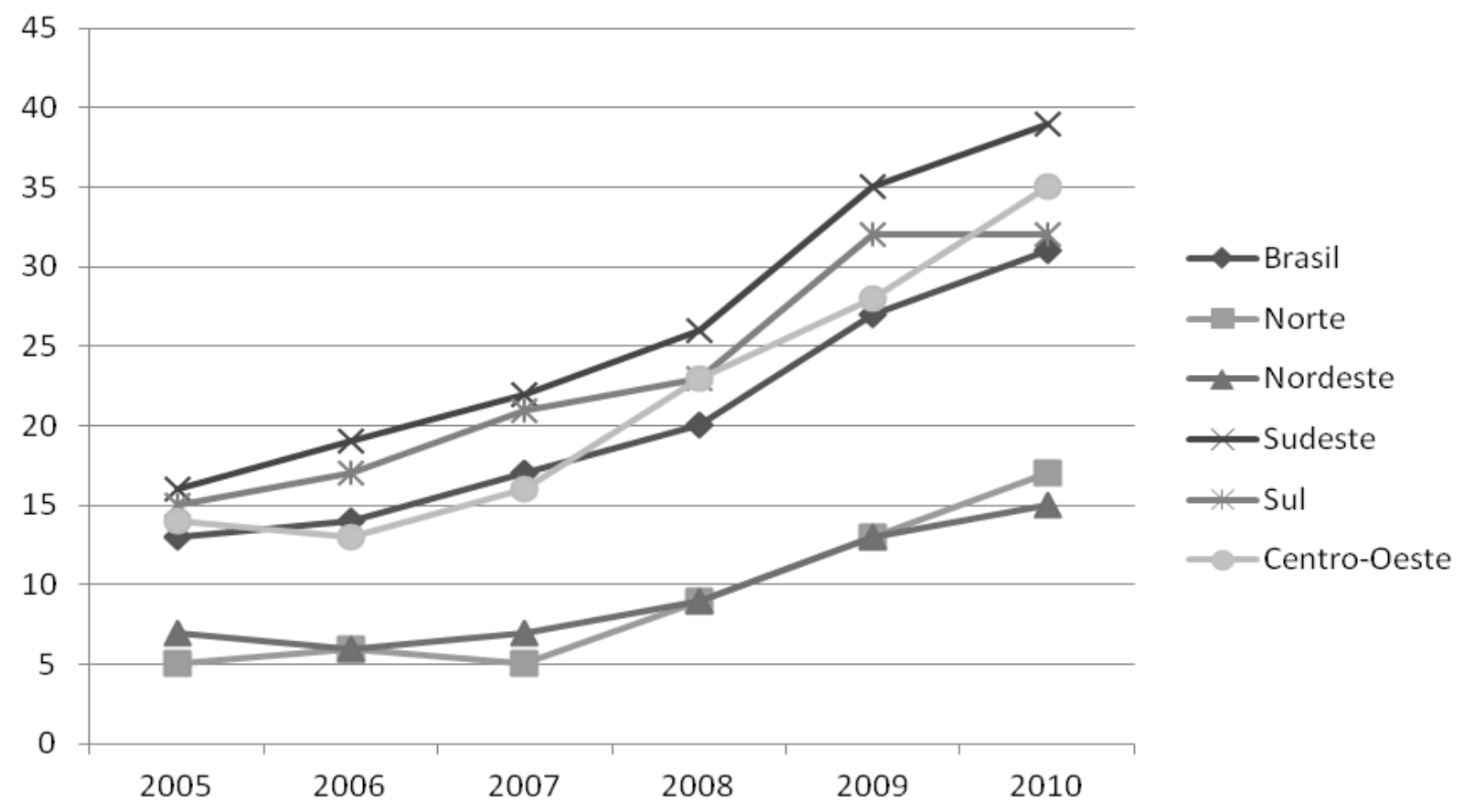

Fonte: Centro de Estudos sobre as Tecnologias da Informação e da Comunicação (2010).

De modo geral, ao se comparar os dados relativos à proporção dos domicílios brasileiros com acesso à internet entre 2005 e 2010, observa-se significativo avanço desta tecnologia nos domicílios do país. Entretanto, o grau de desigualdade entre regiões permanece elevado (Gráfico 1). O Norte e Nordeste concentram as menores taxas, e as regiões Sul, Sudeste e Centro-Oeste apresentam as maiores taxas de domicílios com acesso à internet. Os dados evidenciam, também, que a evolução positiva registrada na região Nordeste foi menor que o avanço registrado nas demais regiões.

Outro aspecto interessante demonstrado pelo levantamento do CETIC.br, é que no ano final da década, o Centro-Oeste ultrapassou a região Sul em termos da proporção de domicílios com acesso à internet. Este resultado pode ser atribuído ao desempenho do Distrito Federal, o que será melhor discutido na seção $\underline{4}$ deste artigo.

O uso do Índice de Inserção dos Estados Brasileiros na Sociedade da Informação e do Conhecimento (IISIC-E) como ferramenta de análise permite demonstrar como que esta desigualdade se revela no âmbito dos estados brasileiros, uma vez que permite classificar as unidades da federação em termos da 
Cecília Araújo Rabelo Corrêa; Elisa Maria Pinto da Rocha; Jane Noronha Carvalhais;

Simone Cristina Dufloth

A sociedade da informação e do conhecimento e os estados brasileiros

sua condição de inserção na SIC. A análise do IISIC-E possibilita, também, identificar fragilidades e potencialidades associadas ao processo de inserção.

\section{METODOLOGIA DE DESENVOLVIMENTO DO ÍNDICE DE ANÁLISE DAS CONDIÇÕES DE INSERÇÃO DOS ESTADOS BRASILEIROS NA SOCIEDADE DA INFORMAÇÃO E DO CONHECIMENTO}

O IISIC-E foi construído a partir do estudo das principais iniciativas internacionais de mensuração da Sociedade da Informação e do Conhecimento (SIC), desenvolvidas por organizações tais como: Rede Iberoamericana de Indicadores de Ciência e Tecnologia (RYCIT), Organização para Cooperação e Desenvolvimento Econômico (OCDE), União Européia/Eurostat, Nações Unidas, Comissão Econômica para América Latina e Caribe (CEPAL) e Observatório da Sociedade da Informação na América Latina e Caribe (OSILAC).

A metodologia sugerida no Manual de Lisboa (LUGONES; SUÁREZ; ALVES, 2009) bem como o trabalho de Araújo (2007), foram referências teóricas e conceituais fundamentais para o desenvolvimento do IISIC-E. A partir das idéias e sugestões neles contidas, buscou-se definir as dimensões para a construção de um índice que permitisse analisar as condições de inserção dos estados brasileiros na SIC.

Embora a idéia inicial da pesquisa fosse construir a Matriz de Indicadores para os estados brasileiros, conforme metodologia sugerida no Manual de Lisboa, a inexistência dos dados estatísticos requeridos para este nível de desagregação (estados da federação) impossibilitou tal empreitada. Diante desta constatação, mostrou-se pertinente, então, o desenvolvimento de uma ferramenta analítica capaz de auxiliar a análise das condições de inserção dos estados brasileiros na SIC que guardasse relação com a base teórica do Manual de Lisboa e para a qual fosse possível a obtenção de dados estatísticos desagregados segundo os estados do País.

O IISIC-E foi construído a partir de quatro (4) dimensões e vinte e nove (29) indicadores que as compõe. De modo geral, pode-se dizer que as dimensões funcionam como os eixos temáticos ou variáveis teóricas e foram definidas tendo como base o estudo da literatura internacional sobre o significado e as medidas de 
Cecília Araújo Rabelo Corrêa; Elisa Maria Pinto da Rocha; Jane Noronha Carvalhais;

Simone Cristina Dufloth

A sociedade da informação e do conhecimento e os estados brasileiros

mensuração voltadas para a SIC, bem como sobre as sugestões para sua adequação à realidade latino-americana. Ou seja, as dimensões representam os fatores relevantes que influenciam o processo de inserção dos estados brasileiros na SIC, e nesse sentido, correspondem a vetores que atuam como fatores que podem fragilizar ou potencializam o processo de inserção.

Conforme mostrado na Figura 2, as dimensões do IISIC-E são: Infraestrutura, Acesso e Uso das Tecnologias da Informação e de Comunicação (posse de telefone, de computador e de computador com acesso a internet nos domicílios do estado; usuários de internet e número de celulares usados pelos habitantes do estado; e presença de banda larga nos municípios do estado), Prioridade Governamental e Governo Eletrônico (alocação de recursos públicos do Governo Estadual na área de ciência, tecnologia e inovação, e escopo do governo eletrônico da administração estadual para interagir com cidadãos e empresas), Base Educacional, Habilidades e Disponibilidade de Recursos Humanos para Sociedade da Informação e do Conhecimento (anos de estudo e nível de formação educacional da população, qualidade do ensino básico, jovens que freqüentam o ensino médio), e Condições Socioeconômicas (presença de serviços de esgotamento sanitário e de coleta de lixo nos domicílios do estado, condições de saúde, ritmo de crescimento da atividade econômica, nível de exportação de produtos intensivos em informação e conhecimento pelas empresas do estado, importância relativa de empresas prestadoras de serviços de informação e comunicação).

Por sua vez, a escolha dos indicadores que retratam as dimensões baseou-se nos seguintes critérios: desagregabilidade territorial dos dados (no nível das unidades da federação), periodicidade anual, proximidade com o conceito abstrato que pretende retratar, simplicidade e confiabilidade das fontes.

A dimensão Infraestrutura, Acesso e Uso das Tecnologias da Informação e das Comunicações expressa a dotação de ferramentas tecnológicas indispensáveis ao desenvolvimento da SIC, bem como aspectos de sua utilização pelas populações que habitam os estados brasileiros. O Manual de Lisboa (RICYT, 2009) sugere que no caso de países latino-americanos e países tecnologicamente menos desenvolvidos, de modo geral, é importante que as condições de infraestrutura tecnológica retratem tecnologias mais complexas como também as tecnologias mais simples (telefone, por exemplo), devido à heterogeneidade das condições reinantes 
Cecília Araújo Rabelo Corrêa; Elisa Maria Pinto da Rocha; Jane Noronha Carvalhais; Simone Cristina Dufloth

A sociedade da informação e do conhecimento e os estados brasileiros

nos países da região, o que também, tornou-se evidente no caso dos estados brasileiros.

A segunda dimensão, Prioridade Governamental e Governo Eletrônico, busca retratar os esforços empreendidos pelos governos estaduais em termos da alocação prioritária de recursos públicos na área de desenvolvimento científico e tecnológico, bem como os esforços no sentido de promover a comunicação com o público-alvo por meio do instrumento de governo eletrônico.

Figura 2 - Dimensões e Indicadores que compõem o Índice de Inserção dos Estados na Sociedade da Informação do Conhecimento (IISIC-E).

\begin{tabular}{|c|c|c|c|}
\hline $\begin{array}{l}\text { Infraestrutura, } \\
\text { Acesso e Uso das } \\
\text { Tecnologias da } \\
\text { Informação e das } \\
\text { Comunicações }\end{array}$ & $\begin{array}{c}\text { Prioridade } \\
\text { Governamental e } \\
\text { Governo } \\
\text { Eletrônico }\end{array}$ & $\begin{array}{c}\text { Base Educacional, } \\
\text { Habilidades e } \\
\text { Disponibilidade de } \\
\text { Recursos } \\
\text { Humanos para } \\
\text { Sociedade da } \\
\text { Informação e do } \\
\text { Conhecimento }\end{array}$ & $\begin{array}{c}\text { Condições } \\
\text { Socioeconômicas }\end{array}$ \\
\hline $\begin{array}{c}\text { Domicílios que } \\
\text { possuem telefone } \\
\text { fixo }\end{array}$ & $\begin{array}{c}\text { Prioridade } \\
\text { governamental e } \\
\text { governo eletrônico }\end{array}$ & $\begin{array}{c}\text { IDEB - Ensino } \\
\text { Médio }\end{array}$ & Crescimento do PIB \\
\hline $\begin{array}{l}\text { Densidade de } \\
\text { celulares }\end{array}$ & $\begin{array}{l}\text { Prioridade do } \\
\text { governo estadual } \\
\text { com TI e formação } \\
\text { dos servidores } \\
\text { públicos }\end{array}$ & $\begin{array}{c}\text { Jovens que } \\
\text { freqüentam } \\
\text { estabelecimento de } \\
\text { ensino }\end{array}$ & $\begin{array}{l}\text { Relação entre } \\
\text { rendimentos de } \\
\text { pobres e ricos }\end{array}$ \\
\hline $\begin{array}{c}\text { Domicílios que } \\
\text { possuem } \\
\text { computador }\end{array}$ & $\begin{array}{c}\text { Informações e } \\
\text { serviços disponíveis } \\
\text { para cidadãos no } \\
\text { site do governo } \\
\text { estadual }\end{array}$ & $\begin{array}{l}\text { Jovens que cursam } \\
\text { ensino superior }\end{array}$ & $\begin{array}{l}\text { Empresas } \\
\text { prestadoras de } \\
\text { serviços de } \\
\text { informação e } \\
\text { comunicação }\end{array}$ \\
\hline $\begin{array}{l}\text { Domicílios com } \\
\text { acesso à internet }\end{array}$ & $\begin{array}{c}\text { Informações e } \\
\text { serviços disponíveis } \\
\text { para empresas no } \\
\text { site do governo } \\
\text { estadual }\end{array}$ & Anos de estudo & $\begin{array}{l}\text { Exportação de } \\
\text { produtos intensivos } \\
\text { em informação e } \\
\text { conhecimento }\end{array}$ \\
\hline $\begin{array}{c}\text { Usuários com } \\
\text { acesso à banda } \\
\text { larga }\end{array}$ & $\begin{array}{c}\text { Empresas que usam } \\
\text { a internet para } \\
\text { consultas e buscas } \\
\text { de informações de } \\
\text { e-Gov }\end{array}$ & $\begin{array}{l}\text { Incentivo à } \\
\text { formação } \\
\text { continuada }\end{array}$ & $\begin{array}{c}\text { Domicílios dotados } \\
\text { de esgotamento } \\
\text { sanitário }\end{array}$ \\
\hline $\begin{array}{c}\text { Municípios } \\
\text { atendidos por banda } \\
\text { larga fixa }\end{array}$ & $\begin{array}{c}\text { Empresas que usam } \\
\text { internet para } \\
\text { interagir com } \\
\text { órgãos públicos }\end{array}$ & $\begin{array}{l}\text { Acesso ao portal de } \\
\text { periódicos da } \\
\text { CAPES }\end{array}$ & $\begin{array}{l}\text { Domicílios dotados } \\
\text { de coleta de lixo }\end{array}$ \\
\hline $\begin{array}{l}\text { Usuários de internet } \\
\text { de alta velocidade } \\
\text { Usuários de internet }\end{array}$ & Usuários de e-Gov & $\begin{array}{c}\text { Pesquisadores com } \\
\text { elevada } \\
\text { qualificação }\end{array}$ & Esperança de vida \\
\hline
\end{tabular}

Fonte: Corrêa (2011, p. 67). 
Cecília Araújo Rabelo Corrêa; Elisa Maria Pinto da Rocha; Jane Noronha Carvalhais;

Simone Cristina Dufloth

A sociedade da informação e do conhecimento e os estados brasileiros

A terceira dimensão corresponde à Base Educacional, Habilidades e Disponibilidade de Recursos Humanos para Sociedade da Informação e do Conhecimento. A educação é reconhecidamente um dos pilares da sociedade que ora se configura, podendo-se dizer que ela é um dos pilares básicos da matriz sugerida pelo Manual de Lisboa. Esta dimensão retrata a capacidade das populações dos estados no sentido de aproveitarem eventuais benefícios proporcionados pelas novas tecnologias, com o desenvolvimento de habilidades e competências técnicas requeridas para o adequado uso destas tecnologias.

A quarta dimensão, Condições Socioeconômicas, busca retratar as condições sociais e econômicas reunidas pelos estados e que tendem a influenciar o desenvolvimento e a consolidação da Sociedade da Informação e do Conhecimento.

Pelo seu significado mais amplo, as dimensões Infraestrutura, Acesso e Uso das Tecnologias da Informação e das Comunicações, e Base Educacional, Habilidades e Disponibilidade de Recursos Humanos corresponderiam a fatores habilitadores cuja presença é essencial para alavancar o processo de inserção dos estados na SIC. Por seu turno, as dimensões Prioridade Governamental e Governo Eletrônico e Condições Socioeconômicas, corresponderiam a fatores que influenciam a capacidade da sociedade, do governo e das empresas para usufruírem dos benefícios proporcionados pelas transformações no padrão tecnoeconômico, mas são fatores suficientes para assegurar condições de inserção favoráveis.

Uma vez definidas as dimensões e indicadores, a obtenção do IISIC-E envolveu a padronização de variáveis, procedimento para no qual aplicou-se o método genebrino de padronização das variáveis, de forma a obter valores comparáveis para os vinte e nove indicadores. Assim, os indicadores padronizados assumem valores que variam no intervalo entre "zero" (0) e "um" (1), sendo que, quanto mais próximo da unidade (1), mais favorável é a posição daquele estado em relação aos outros. Os sub-índices das dimensões correspondem ao valor médio dos indicadores padronizados de cada dimensão, de forma que a partir dos valores obtidos para os sub-índices torna-se possível, então, verificar a posição relativa dos estados no que diz respeito a cada dimensão.

Por sua vez, o índice geral, ou seja, o indicador sintético denominado Índice das Condições de Inserção dos Estados na Sociedade da Informação e do 
Cecília Araújo Rabelo Corrêa; Elisa Maria Pinto da Rocha; Jane Noronha Carvalhais;

Conhecimento (IISIC-E) corresponde, então, ao valor médio dos sub-índices das dimensões. Quanto mais elevado (mais próximo de "um", 1) for o valor do IISIC-E, mais favoráveis tendem a ser as condições de inserção do estado; e quanto menores (mais próximos de "zero") menos favoráveis tendem a ser as condições de inserção, possibilitando assim, a classificação dos estados em termos das condições por eles reunidas em termos de sua inserção no novo padrão tecnoeconômico.

As quatro categorias e os parâmetros utilizados para a classificação dos estados brasileiros em relação às condições por eles reunidas para inserir-se na SIC são mostrados no Quadro 1.

Além da comparação dos valores do IISIC-E entre os estados brasileiros, outro recorte analítico interessante diz respeito à comparação dos valores obtidos para os sub-índices de cada dimensão para os estados, o que permite identificar as principais fragilidades que se apresentariam aos estados classificados nas Categorias $\mathrm{C}$ e D (condições de inserção altamente desfavoráveis e desfavoráveis). Este aspecto é importante, pois sinaliza para fatores a serem priorizados pelas políticas públicas voltadas para a promoção de melhores condições de inserção dos estados no novo padrão tecnoeconômico de produção.

Quadro 1 - Categorias de Condições de Inserção dos Estados Brasileiros.

\begin{tabular}{|c|l|c|}
\hline Categoria & \multicolumn{1}{|c|}{ Nome } & Valor do IISIC-E \\
\hline A & $\begin{array}{l}\text { Estados que reúnem condições de inserção altamente } \\
\text { favoráveis }\end{array}$ & Acima de 0,75 \\
\hline B & Estados que reúnem condições de inserção favoráveis & Entre 0,5 e 0,74 \\
\hline C & Estados que reúnem condições de inserção desfavoráveis & Entre 0,25 e 0,49 \\
\hline D & $\begin{array}{l}\text { Estados que reúnem condições de inserção altamente } \\
\text { desfavoráveis }\end{array}$ & Menos de 0,24 \\
\hline
\end{tabular}

Fonte: Corrêa (2011, p.106).

4 ANÁLISE DAS CONDIÇÕES DE INSERÇÃO DOS ESTADOS BRASILEIROS NA SOCIEDADE DA INFORMAÇÃO E DO CONHECIMENTO 
Cecília Araújo Rabelo Corrêa; Elisa Maria Pinto da Rocha; Jane Noronha Carvalhais; Simone Cristina Dufloth

A sociedade da informação e do conhecimento e os estados brasileiros

Nesta seção são analisados os principais resultados da pesquisa, a partir dos valores obtidos para o índice geral e para os sub-índices (ou dimensões), buscandose classificar e comparar a posição relativa dos estados brasileiros, bem como identificar as principais fragilidades que se apresentam aos estados em seu processo de inserção na SIC.

Os valores encontrados para os estados brasileiros em 2010 são mostrados no Gráfico 2, e confirmam a idéia geral sobre a heterogeneidade e disparidade existente nas condições de inserção entre os estados brasileiros. Os valores obtidos para o IISIC-E variaram do extremo superior de 0,76 ao extremo inferior 0,15 , sendo o maior valor registrado para o Distrito Federal, e o menor para o Estado do Maranhão. Nesse sentido, os resultados da pesquisa indicam que o primeiro estado reúne condições de inserção altamente favoráveis enquanto Maranhão representaria o extremo oposto (condições altamente desfavoráveis).

O segundo aspecto importante revelado pelo estudo diz respeito ao fato de vinte e um (dentre os vinte e sete estados) se classificarem nas categorias $C$ e $D$, indicando que a grande maioria dos estados brasileiros $(77,8 \%)$ reúne condições de inserção desfavoráveis. Considerando-se este grupo de estados (categorias $C$ e D), - Maranhão e Piauí são os estados correspondem ao caso extremo, reunindo condições de inserção altamente desfavoráveis. Portanto, uma minoria de estados $(22,2 \%)$ apresenta condições de inserção favoráveis, sendo o Distrito Federal o único a reunir condições de inserção altamente favoráveis, seguido por São Paulo, Paraná, Santa Catarina, Rio Grande do Sul e Rio de Janeiro classificados na categoria B (condições favoráveis), conforme mostrado no Gráfico 2.

Nesse sentido, a pesquisa corrobora, também, os levantamentos realizados pelo CETIC.br no âmbito das grandes regiões do País e que, conforme discutido na seção $\underline{2}$, apontaram o Centro-Oeste como a região brasileira que apresenta a maior proporção de domicílios com acesso à internet, e o Nordeste como a região em que esta proporção registra o menor valor (Gráfico 1 e 2). 
Cecília Araújo Rabelo Corrêa; Elisa Maria Pinto da Rocha; Jane Noronha Carvalhais; Simone Cristina Dufloth

A sociedade da informação e do conhecimento e os estados brasileiros

Gráfico 2 - Resultados do Índice de Inserção dos Estados na Sociedade da Informação e do Conhecimento (IISIC-E)

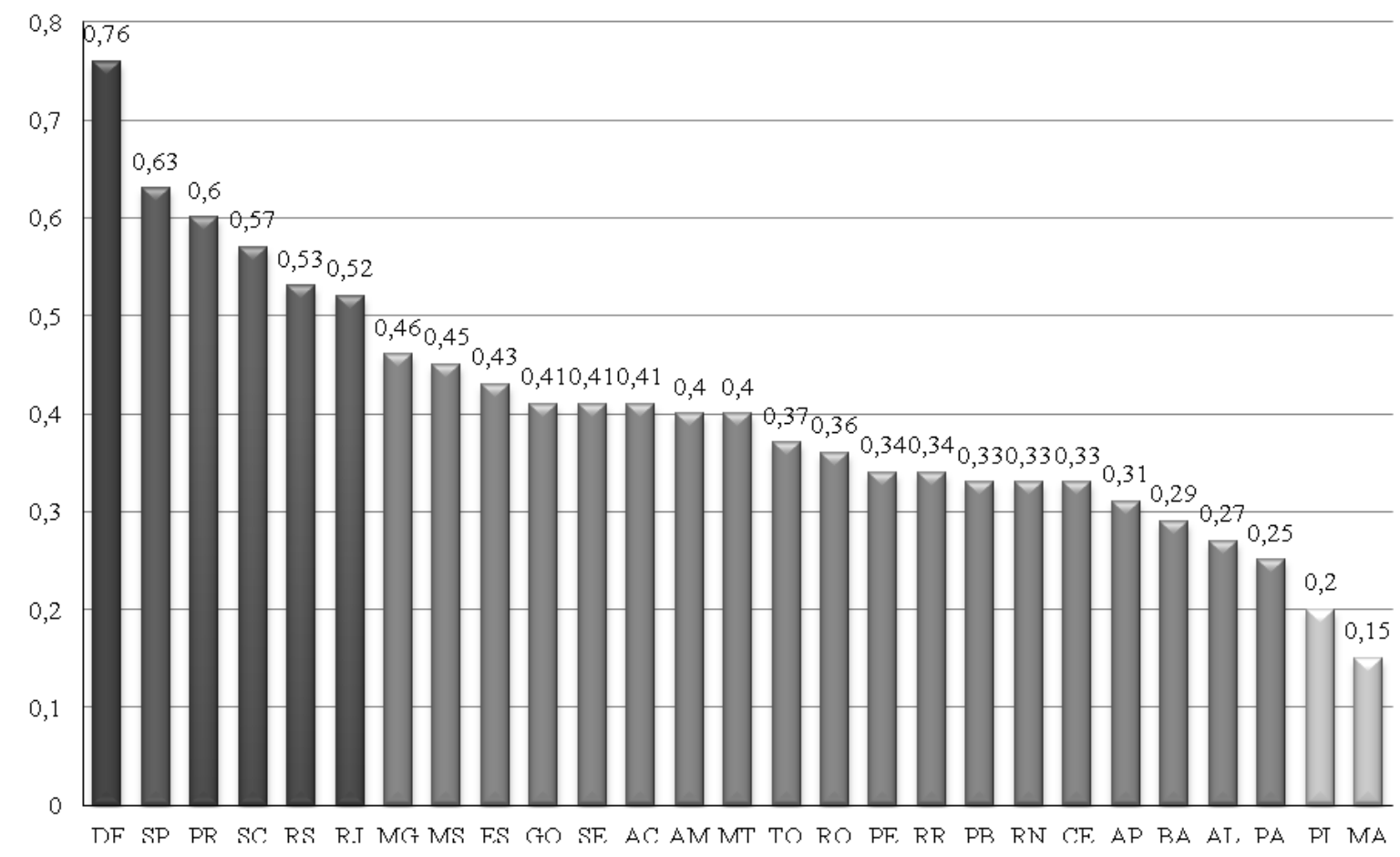

Fonte: Corrêa (2011, p. 107).

No que tange ao Distrito Federal - única unidade da federação posicionada na categoria A - chama a atenção o valor do sub-índice da dimensão Infraestrutura, Acesso e Uso de Tecnologias da Informação e das Comunicações, que alcançou o maior nível valor possível, "um" (1), bem como aquele registrado para o sub-índice correspondente a Prioridade Governamental e Governo Eletrônico $(0,55)$. Tendência semelhante foi registrada para a maioria dos estados classificados na categoria $B$ (condições de inserção favoráveis). Este resultado sugere que, conforme esperado, a dotação de infraestrutura tecnológica e as condições de acesso e uso das tecnologias da informação pela população dos estados, apresenta-se como fator que habilita a inserção na Sociedade da Informação e do Conhecimento. A dimensão Prioridade Governamental e Governo Eletrônico, por seu turno, retrataria condições capacitadoras relevantes, embora não suficientes para assegurar a existência de condições de inserção favoráveis (Tabela 1).

Outro aspecto que chama a atenção é a posição ocupada pelos estados de Minas Gerais e Espírito Santo (IISIC-E igual a 0,46 e 0,43, respectivamente). Embora ambos integrem a região Sudeste - reconhecidamente aquela de maior 
Cecília Araújo Rabelo Corrêa; Elisa Maria Pinto da Rocha; Jane Noronha Carvalhais;

Simone Cristina Dufloth

A sociedade da informação e do conhecimento e os estados brasileiros

capacidade econômica de produção do País - eles classificam-se na categoria C (condições de inserção desfavoráveis).

Tabela 1 - Índice das Condições de Inserção dos Estados na Sociedade da Informação e do Conhecimento e Valor dos Sub-Índices Correspondentes às Dimensões (em ordem decrescente do valor do IISIC-E obtido para os estados).

\begin{tabular}{|c|c|c|c|c|c|}
\hline \multirow[b]{2}{*}{$\mathbf{U F}$} & \multirow[b]{2}{*}{ IISIC-E } & \multicolumn{4}{|c|}{ Valores das Dimensões do IISIC-E } \\
\hline & & $\begin{array}{c}\text { Infraestrutura, } \\
\text { Acesso e Uso } \\
\text { das TICs }\end{array}$ & $\begin{array}{c}\text { Prioridade } \\
\text { Governamental } \\
\text { e Governo } \\
\text { Eletrônico }\end{array}$ & $\begin{array}{c}\text { Base Educacional, } \\
\text { Habilidades e } \\
\text { Disponibilidade } \\
\text { de Recursos } \\
\text { Humanos para } \\
\text { SIC }\end{array}$ & $\begin{array}{c}\text { Condições } \\
\text { Socioeconômicas }\end{array}$ \\
\hline DF & 0,76 & 1,00 & 0,55 & 0,83 & 0,68 \\
\hline SP & 0,63 & 0,77 & 0,43 & 0,59 & 0,72 \\
\hline $\mathbf{P R}$ & 0,60 & 0,64 & 0,44 & 0,63 & 0,69 \\
\hline SC & 0,57 & 0,66 & 0,32 & 0,64 & 0,64 \\
\hline $\mathbf{R S}$ & 0,53 & 0,53 & 0,27 & 0,73 & 0,60 \\
\hline RJ & 0,52 & 0,66 & 0,23 & 0,64 & 0,55 \\
\hline MG & 0,46 & 0,49 & 0,30 & 0,47 & 0,58 \\
\hline MS & 0,45 & 0,49 & 0,32 & 0,48 & 0,49 \\
\hline $\mathbf{E S}$ & 0,43 & 0,54 & 0,35 & 0,35 & 0,48 \\
\hline GO & 0,41 & 0,43 & 0,43 & 0,37 & 0,43 \\
\hline SE & 0,41 & 0,31 & 0,49 & 0,35 & 0,50 \\
\hline $\mathbf{A C}$ & 0,41 & 0,31 & 0,69 & 0,31 & 0,33 \\
\hline $\mathbf{A M}$ & 0,40 & 0,19 & 0,63 & 0,37 & 0,41 \\
\hline MT & 0,40 & 0,42 & 0,39 & 0,39 & 0,38 \\
\hline TO & 0,37 & 0,25 & 0,43 & 0,30 & 0,40 \\
\hline RO & 0,36 & 0,17 & 0,45 & 0,35 & 0,30 \\
\hline PE & 0,34 & 0,30 & 0,38 & 0,29 & 0,39 \\
\hline RR & 0,34 & 0,25 & 0,38 & 0,49 & 0,31 \\
\hline PB & 0,33 & 0,18 & 0,33 & 0,46 & 0,37 \\
\hline $\mathbf{R N}$ & 0,33 & 0,24 & 0,34 & 0,39 & 0,34 \\
\hline $\mathbf{C E}$ & 0,33 & 0,27 & 0,39 & 0,28 & 0,36 \\
\hline $\mathbf{A P}$ & 0,31 & 0,19 & 0,38 & 0,28 & 0,37 \\
\hline $\mathbf{B A}$ & 0,29 & 0,33 & 0,23 & 0,22 & 0,39 \\
\hline $\mathbf{A L}$ & 0,27 & 0,21 & 0,36 & 0,20 & 0,30 \\
\hline $\mathbf{P A}$ & 0,25 & 0,12 & 0,38 & 0,19 & 0,30 \\
\hline PI & 0,20 & 0,18 & 0,27 & 0,23 & 0,15 \\
\hline MA & 0,15 & 0,10 & 0,18 & 0,11 & 0,22 \\
\hline
\end{tabular}

Fonte: Corrêa (2011, p. 117).

Ainda a este respeito, os resultados obtidos para o Estado de Minas Gerais e Bahia ilustram com bastante propriedade este argumento. Ao comparar os valores 
Cecília Araújo Rabelo Corrêa; Elisa Maria Pinto da Rocha; Jane Noronha Carvalhais;

Simone Cristina Dufloth

A sociedade da informação e do conhecimento e os estados brasileiros

dos sub-índices das quatro dimensões para cada estado, o maior valor observado entre elas corresponde às Condições Socioeconômicas $(0,58$ no caso de Minas Gerais e 0,39 para a Bahia). Entretanto, na classificação geral do IISIC-E, tanto o estado de Minas Gerais quanto o da Bahia detêm posição desconfortável em termos das condições de inserção na SIC, classificando-se na categoria C (Gráfico 2, Tabela 1).

Portanto, fatores outros que não aqueles preponderantemente econômicos influenciam as condições de inserção dos estados na Sociedade da Informação e do Conhecimento, aspecto que deve merecer a atenção dos formuladores da política macroeconômica do País. Excessivamente focada no tripé inflação-juros-câmbio ou seja, nas políticas inflacionária, monetária e cambial - a política macroeconômica brasileira acaba tomando como secundárias políticas e programas públicos a serem formulados no âmbito das políticas educacional, industrial e de comércio exterior.

Outro resultado interessante que se apreende do estudo diz respeito ao enfoque da análise nos dois principais grupos de estados: o primeiro, reunindo os estados classificados nas categorias C e D (ou seja, no conjunto de estados que apresentaram condições de inserção desfavoráveis); e o segundo grupo, composto pelos estados classificados nas categorias A e B (conjunto de estados que apresentaram condições de inserção favoráveis). A obtenção do valor médio dos sub-índices das dimensões para cada grupo de estados (procedimento realizado por meio dos dados mostrados na Tabela 1), possibilita inferências sobre as dimensões mais frágeis do processo de inserção dos estados na SIC.

No caso do primeiro grupo (estados que reuniram condições de inserção desfavoráveis), o valor médio dos sub-índices correspondentes às dimensões é o seguinte: Infraestrutura, Acesso e Uso das TIC, 0,284; Prioridade Governamental e Governo Eletrônico, 0,386; Habilidades e Disponibilidade de Recursos Humanos, 0,328; e Condições Socioeconômicas, 0,371. Para o segundo grupo (estados que apresentaram condições de inserção favoráveis, os valores são: Infraestrutura, Acesso e Uso das TIC, 0,710; Prioridade Governamental e Governo Eletrônico, 0,373; Habilidades e Disponibilidade de Recursos Humanos, 0,677; e Condições Socioeconômicas, 0,647.

Quanto a estes dados, pelo menos dois aspectos principais merecem ser destacados. Primeiramente, observa-se que no caso do primeiro grupo (estados que 
Cecília Araújo Rabelo Corrêa; Elisa Maria Pinto da Rocha; Jane Noronha Carvalhais;

Simone Cristina Dufloth

A sociedade da informação e do conhecimento e os estados brasileiros

apresentaram condições de inserção desfavoráveis), os menores valores correspondem às dimensões Infraestrutura, Acesso e Uso das $\operatorname{TIC}(0,284)$, e Habilidades e Disponibilidade de Recursos Humanos para a SIC $(0,328)$. Para o segundo grupo (estados que apresentaram condições de inserção favoráveis), o menor valor corresponde à dimensão Prioridade Governamental e Governo Eletrônico $(0,379)$. Assim, os resultados sugerem que os principais pontos fracos ou fragilidades que se impõem ao processo de inserção dos estados cujas condições de inserção se mostram desfavoráveis são, principalmente: carências e insuficiências de infraestrutura tecnológica que limitam o acesso e o uso das novas tecnologias pela população; inadequações e baixa disponibilidade de recursos humanos com nível educacional compatível com os requerimentos de qualificação exigidos pelo novo padrão tecnoeconômico de produção.

O outro ponto interessante revelado pela pesquisa diz respeito diz à dimensão Prioridade Governamental e Governo Eletrônico, que parece atuar como fator capacitador das condições de inserção no caso dos estados que apresentaram condições de inserção favoráveis (categorias C e D). Ou seja, para o grupo de estados que já reúne condições de inserção favoráveis, os resultados sinalizam para a importância da atuação dos governos estaduais no sentido de priorizar a alocação de recursos públicos na área de ciência e tecnologia.

Diante das fragilidades identificadas e considerando as políticas públicas voltadas para a promoção de ambiente favorável à inserção mais homogênea dos estados no novo padrão tecnoeconômico - e que no caso brasileiro se encontram sob a competência do Ministério da Ciência, Tecnologia e Inovação, principalmente - torna-se fundamental a formulação conjunta de programas e projetos públicos capazes de aprofundar a sinergia entre a política nacional de ciência e tecnologia, a política educacional e a industrial.

Portanto, o esforço governamental para que o conjunto dos estados brasileiros - e não apenas uma minoria deles - consiga reunir condições de usufruir dos benefícios decorrentes de sua inserção na SIC - requer maior interação entre distintos ministérios e órgãos públicos. E esta interação representa grande desafio para as políticas públicas no Brasil, cujo processo de formulação é reconhecidamente caracterizado pela excessiva fragmentação de iniciativas, medidas, programas e públicos. 
Cecília Araújo Rabelo Corrêa; Elisa Maria Pinto da Rocha; Jane Noronha Carvalhais;

Simone Cristina Dufloth

A sociedade da informação e do conhecimento e os estados brasileiros

\section{CONSIDERAÇÕES FINAIS}

O Brasil vivencia um momento no qual se encontra em formação um novo padrão de acumulação capitalista, caracterizado pela crescente importância que a informação, o conhecimento e as inovações tecnológicas passam a assumir na configuração deste padrão.

A idéia central é que a análise das condições de inserção dos estados brasileiros neste padrão tecnoeconômico, objeto deste estudo, possa contribuir para o aperfeiçoamento das políticas públicas voltadas para a consolidação de uma Sociedade da Informação mais homogênea, em que os estados brasileiros, em seu conjunto, possam usufruir dos benefícios possibilitados pelo padrão de produção ora em formação.

Os resultados da pesquisa evidenciam a necessidade de intervenção do Estado no sentido de promover a construção de ambiente mais igualitário no que diz respeito ao processo de inserção dos estados no novo padrão tecnoeconômico de produção, uma vez que a grande maioria das unidades da federação ainda não reúne condições que favoreçam a sua inserção na SIC.

A título de síntese, pelo menos três outras considerações finais mostram-se relevantes.

$\mathrm{Na}$ atual fase da política de ciência e tecnologia, os principais programas e projetos públicos são conduzidos no âmbito da esfera do Governo Federal, e traduzem o esforço governamental na universalização de infraestrutura tecnológica e serviços a ela associados, podendo-se citar: programa de inclusão digital voltado para a criação de telecentros, centros vocacionais tecnológicos, incentivo ao uso de computadores e internet nas escolas públicas, e plano nacional de banda larga, dentre outras iniciativas. Conforme evidenciado pela pesquisa, uma das principais fragilidades do processo de inserção dos estados na SIC é justamente a carência e insuficiência da infraestrutura tecnológica de apoio à disseminação das novas tecnologias, podendo-se dizer, então, que a política de universalização do Governo Federal está no caminho correto. Entretanto, ela não se mostra suficiente e sua eficácia parece depender, crescentemente, de uma atuação conjunta do Ministério da Ciência, Tecnologia e Inovação com outros ministérios setoriais e órgãos públicos. 
Cecília Araújo Rabelo Corrêa; Elisa Maria Pinto da Rocha; Jane Noronha Carvalhais;

Simone Cristina Dufloth

A sociedade da informação e do conhecimento e os estados brasileiros

O estudo aponta a importância dos Governos Estaduais ampliarem e priorizarem iniciativas e programas voltados para o desenvolvimento científico e tecnológico. Como já ocorre em outras áreas (como saúde, por exemplo), aqui também parece interessante a adoção de mecanismos nacionais de indução e estímulo à participação dos Governos Estaduais.

Finalmente, a construção de um ambiente mais homogêneo favorável à inserção dos estados na SIC requer iniciativas outras que não apenas aquelas de natureza preponderantemente econômica.

\section{REFERÊNCIAS}

ARAÚJO, Evandro Nicomedes. Sociedade da informação no Brasil: uma proposta de mensuração e sua aplicação para o período 2001-2004. 2007. Dissertação (Mestrado em Administração Pública) - Escola de Governo da Fundação João Pinheiro, Belo Horizonte, 2007.

CENTRO DE ESTUDOS SOBRE AS TECNOLOGIAS DA INFORMAÇÃO E DA COMUNICAÇÃO - CETIC.br. Pesquisas e indicadores. 2010. Disponível em: <http://www.CETIC.br/pesquisas-indicadores.htm >. Acesso em: 10 abr. 2012.

COMISSÃO ECONÔMICA PARA AMÉRICA LATINA E CARIBE - CEPAL. Hacia un plan de Acción de América Latina y el Caribe para la Sociedad de la Información eLAC 2007. CEPAL, 2007. Disponível em: <http://www.elac2007.info>. Acesso em: 12 nov. 2011.

CORRÊA, Cecília Araújo Rabelo. Sociedade da informação e do conhecimento: análise das condições de inserção dos estados brasileiros. 2011. Dissertação (Mestrado em Administração Pública) - Escola de Governo Professor Paulo Neves Coelho, Fundação João Pinheiro, Belo Horizonte, 2011.

FUNDAÇÃO JOÃO PINHEIRO - FJP. Análise das condições de inserção dos estados brasileiros na sociedade da informação e do conhecimento: proposta metodológica e aplicação para as unidades da federação. Belo Horizonte: Fundação João Pinheiro, 2010. Texto para discussão n.1.

LUGONES, Gustavo (Org.). Indicadores de la sociedad del conocimiento: aspectos conceptuales y metodológicos. Buenos Aires: Centro de Estudios sobre Ciencia-Desarrollo y Educación Superior. 2002. Documento de Trabajo n².

LUGONES, Gustavo; SUÁREZ, Diana; ALVES, Nuno de Almeida (Coord.). Manual de Lisboa: orientações para a interpretação dos dados estatísticos disponíveis e para a construção de indicadores referentes à transição da América Latina para a sociedade da informação. Lisboa: RICYT, 2009. 
Cecília Araújo Rabelo Corrêa; Elisa Maria Pinto da Rocha; Jane Noronha Carvalhais; Simone Cristina Dufloth

A sociedade da informação e do conhecimento e os estados brasileiros

NERI, Marcelo Côrtes (Coord.). Mapa da exclusão digital. Rio de Janeiro: FGV/IBRE, CPS, 2003.

OBSERVATORY FOR THE INFORMATION SOCIETY IN LATIN AMERICA AND THE CARIBBE - OSILAC. Benchmarking the plan of action of the world summit on the information society (WSIS) in Latin America and the Caribbean. Santiago of Chile, 2005. (Version 3.0).

ORGANIZAÇÃO PARA A COOPERAÇÃO E DESENVOLVIMENTO ECONÔMICO OCDE. Guide to measuring the information society. Paris: OCDE, 2007.

ORGANIZAÇÃO PARA A COOPERAÇÃO E DESENVOLVIMENTO ECONÔMICO OCDE. Core set of indicators for environmental performance reviews: a synthesis report by the group on the state of the environment. Paris: OCDE, 1993.

ORGANIZAÇÃO PARA A COOPERAÇÃO E DESENVOLVIMENTO ECONÔMICO OCDE. Oslo manual: OECD proposed guidelines for collecting and interpreting technological innovation data. Paris, 1997.

ROCHA, Elisa Maria Pinto. Indicadores de inovação: uma proposta a partir da perspectiva da informação e do conhecimento. 2003. 264f. Tese (Doutorado em Ciência da Informação) - Escola de Ciência da Informação, UFMG, Belo Horizonte, 2003.

SCANDAR NETO, Wadih João, JANNUZZI, Paulo de Martino; SILVA, Pedro Luis do Nascimento. Sistemas de indicadores ou indicadores sintéticos: do que precisam os gestores de programas sociais? In: ENCONTRO NACIONAL DE ESTUDOS POPULACIONAIS, 16., 2008, Caxambu. Anais... Caxambu, 2008.

Title

The information and knowledge society and the brazilian states

\section{Abstract}

Introduction: The term Information Society and Knowledge is used to characterize the new patterns of capital accumulation that assumes greater importance with the $90 \mathrm{~s}$. In this new technological and productive standard, information, knowledge and technological innovations become determinants of socio-economic development of a nation. Brazil has a great internal inequality which tends to influence the conditions of insertion of the Brazilian states in this society.

Objective: The aim of this paper is to analyze the conditions of insertion of the Brazilian states in the techno-productive standard now taking shape in Brazil and worldwide.

Methodology: Literature Review and Document Analysis.

Results: The results support the general idea about the about the heterogeneity of the conditions of participation of states in the new technological and economic pattern. The vast majority of states had insertion unfavorable conditions, and dimensions that explain this 
Cecília Araújo Rabelo Corrêa; Elisa Maria Pinto da Rocha; Jane Noronha Carvalhais;

Simone Cristina Dufloth

A sociedade da informação e do conhecimento e os estados brasileiros

uncomfortable position are: Infrastructure, Access and Use of Information Technologies and Communications, and Skills and Availability of Human Resources.

Conclusion: The study suggest that advances in building favorable insertion states depend basically on the adoption of measures to deepen the synergy between science and technology policy, education and industrial policy.

Keywords: Information and knowledge society. Conditions for insertion of the states. Measurement indicators.

\section{Título}

La Sociedad de la Información y el Conocimiento y los Estados de Brasil

\section{Resumen}

Introducción: El término Sociedad de la Información y el Conocimiento se utiliza para caracterizar los nuevos patrones de acumulación de capital que adquiere mayor importancia con los años 90 . En esta nueva norma tecnológica y productiva, información, conocimientos, innovaciones y tecnológicos se vuelven determinantes de desarrollo socio-económico de una nación. Brasil tiene una gran desigualdad interna que tiende a influir en las condiciones de inserción de los estados brasileños de esta sociedad.

Objetivo: El objetivo de este trabajo es analizar las condiciones de inserción de los estados brasileños en el nivel tecno-productivo tomando forma en Brasil y en todo el mundo.

Metodología: Revisión de la literatura y análisis documental.

Resultados: Los resultados apoyan la idea general acerca de la heterogeneidad de las condiciones de participación de los estados en el nuevo patrón tecnológico y económico. La gran mayoría de los estados tienen condiciones desfavorables de inserción, y las dimensiones que explican esta incómoda posición son: Infraestructura, Acceso y Uso de Tecnologías de la Información y las Comunicaciones, y de las capacidades y la disponibilidad de recursos humanos.

Conclusiones: El estudio sugiere que los avances en la construcción de los estados favorables de inserción dependen básicamente de la adopción de medidas para profundizar la sinergia entre la política científica y tecnologica, la política educativa y industrial.

Palabras clave: Sociedad de la información y del conocimiento. Condiciones para la inserción de los estados. Medición de indicadores.

Recebido em: 05.06.2012

Aceito em: 20.09.2013 